\title{
Electron Microscopy in the Context of a Children's Research Hospital
}

\section{Cam Robinson}

\section{St. Jude Children's Research Hospital, Memphis, Tennessee, United States}

An electron microscopy lab is unlikely to be the first thing that one associates with a research hospital, especially one that is nationally known for focusing on catastrophic childhood diseases, notably cancers and blood disorders. In a world of "-omics", where cancers are defined by their genomes and protein or metabolic expression profiles, where does traditional electron microscopy, let alone FIB-SEM 3D volume imaging and electron tomography, fit into a research hospital and how is it providing researchers needed information in the fight against these devastating diseases?

The Electron Microscopy division of the Cell and Tissue Imaging Center at St. Jude Children's Research Hospital provides ultrastructural imaging services to the St. Jude research community, servicing a variety of needs. One of the primary responsibilities is confirming or providing morphological descriptors for orthotropic xenografts. In many cases, these come to the electron microscopy lab having been extensively characterized by other means before they are imaged. The ultrastructural imaging serves to confirm that the expected morphological hallmarks ${ }^{1,2}$, some which are only observable at the electron microscope, are present. However, in a subset of these samples, the ultrastructural characterization does not match to the expected tumor type or, in some cases, there are no established ultrastructural characteristics for that particular tumor type. For these cases, the electron microscopy provides important insights based off of the characteristics back to our researchers. Additionally, electron micrographs are provided for all xenograft samples made available to the larger cancer research community via St. Jude's Childhood Solid Tumor Network $^{3}$. Similarly, the Center also sees samples originating from the research laboratories in which an investigator has induced a perturbation, often in the dissection of various cellular pathways that are known or thought to go awry in cancer or other developmental processes, that results in an unexpected phenotype and they look to the Center to provide ultrastructural morphology in support of modeling these perturbations and their effect at the cellular to sub-cellular level.

In addition to other projects where an investigator simply needs the resolving power of the electron microscope to observe their organelle or sub-cellular interaction of interest, the Center provides 3D volumes by FIB-SEM, large area acquisitions, and 2D/3D correlative light and electron microscopy services. These are often instrumental in placing a particular morphology in the larger context of the cell or tissue. As an example, large area acquisitions have been instrumental in the characterization of conditions resulting in tissue mosaics. In the case of FIB-SEM 3D volumes, these have covered a wide range of samples in which cellular context have been vital to the full understanding of the disease process. Where this is combined with light level imaging, the ability to correlate a variety of immunofluorescent or genetically induced fluorescent markers allows for a more precise identification of the ultrastructural correlates related to these markers.

In an environment such as the St. Jude research community, one of the notable challenges is dealing with investigators that request ultrastructural characterization of a unique cell population. Often these are a selected population of cells from a hematopoietic lineage and the sample can be surprisingly small both in available cell number and cell size. Although electron microscopists often deal with small samples, working with a vanishingly small population of cells is a continual challenge and has required the development of specific workflows to ensure successful processing of such samples. 
The ultrastructural examination of samples adds a significant piece in the investigation into childhood and pediatric cancers, even in in an environment already rich in genomic, proteomic, metabolomic and other characterizations. $^{4}$

\section{References}

1 Kim, N. R., Ha, S. Y. \& Cho, H. Y. Utility of transmission electron microscopy in small round cell tumors. J Pathol Transl Med 49, 93-101, doi:10.4132/jptm.2015.01.30 (2015).

2 Hicks, J. \& Mierau, G. W. The spectrum of pediatric tumors in infancy, childhood, and adolescence: a comprehensive review with emphasis on special techniques in diagnosis. Ultrastruct Pathol 29, 175202, doi:10.1080/01913120590951185 (2005).

3 Stewart, E. et al. Orthotopic patient-derived xenografts of paediatric solid tumours. Nature 549, 96-100, doi:10.1038/nature23647 (2017).

$4 \quad$ The Cell and Tissue Imaging Center is supported by NCI P30 CA021765 and St. Jude Children's Research Hospital. 